\title{
Eficácia do emprego do espelho de Glatzel na avaliação da permeabilidade nasal
}

\section{Efficacy of the use of the Glatzel mirror in nasal permeability evaluation}

\author{
Iara Barreto Bassi' ${ }^{1}$, Letícia Paiva Franco ${ }^{2}$, Andréa Rodrigues Motta ${ }^{3}$
}

\begin{abstract}
RESUMO
Objetivo: Comparar a medida do escape aéreo nasal, realizada por meio espelho de Glatzel, entre crianças com e sem obstrução de via aérea superior, de acordo com o sexo e a faixa etária. Métodos: Amostra composta por 43 crianças, com média de 6,9 anos, separadas em quatro grupos: com indicação cirúrgica para desobstrução de via aérea superior (grupo cirúrgico), alérgicos com manifestação de obstrução (grupo alérgico), alérgicos com obstrução e indicação de cirurgia (grupo alérgico e cirúrgico) e crianças sem obstrução (grupo controle). A medida do escape foi realizada por meio do Espelho Nasal Milimetrado de Altmann e os dados mensurados em $\mathrm{cm}^{2}$ pelo software AutoCAD 2002. Os dados foram analisados por meio de estatística descritiva e por testes de hipótese com nível de significância de 5\%. Resultados: $\mathrm{O}$ grupo controle foi o que apresentou a maior média $\left(6,53 \mathrm{~cm}^{2}\right)$ na medida do escape aéreo nasal, enquanto o grupo alérgico e cirúrgico apresentou a menor $\left(3,07 \mathrm{~cm}^{2}\right)$. As médias dos grupos alérgico e cirúrgico foram de $4,74 \mathrm{~cm}^{2}$ e $5,91 \mathrm{~cm}^{2}$, respectivamente. Apenas o grupo alérgico e cirúrgico apresentou diferença significante em relação ao grupo controle. Verificou-se ainda que a medida de escape não foi estatisticamente afetada pelas variáveis sexo e idade. Conclusão: O espelho de Glatzel demonstrou não ser um instrumento confiável para a avaliação da permeabilidade nasal na amostra investigada, exceto nos casos de grande obstrução nasal, ou seja, pacientes cirúrgicos e também alérgicos. Não foram observadas correlações com as variáveis: sexo e faixa etária.
\end{abstract}

Descritores: Obstrução nasal; Respiração bucal; Estudos de avaliação; Permeabilidade; Criança

\section{INTRODUÇÃO}

O espelho de Glatzel caracteriza-se como um instrumento objetivo de avaliação da permeabilidade nasal extremamente simples. Esse instrumento consiste em uma placa de metal polida e graduada que, ao ser posicionada sob as narinas, condensa o vapor d'água do ar expirado. A área embaçada é então mensurada e a avaliação da função nasal pode ser realizada ${ }^{(1)}$.

$\mathrm{O}$ instrumento pode ser empregado como recurso para

Este artigo é parte de trabalho de conclusão do Curso de Fonoaudiologia da Universidade Federal de Minas Gerais - UFMG - Belo Horizonte (MG), Brasil, disponível em: http://www.medicina.ufmg.br/fon/monografias/07-iara-bassi.pdf (1) Pós-graduanda (Mestrado) em Saúde e Trabalho pelo Programa de Pósgraduação em Saúde Pública do Departamento de Medicina Preventiva e Social da Faculdade de Medicina da Universidade Federal de Minas Gerais - UFMG - Belo Horizonte (MG), Brasil.

(2) Mestre, Professora substituta do Departamento de Oftalmologia e Otorrinolaringologia da Universidade Federal de Minas Gerais - UFMG - Belo Horizonte (MG), Brasil.

(3) Pós-graduanda (Doutorado) em Distúrbios da Comunicação Humana pela Universidade Federal de São Paulo - UNIFESP - São Paulo (SP), Brasil; Professora assistente do Departamento de Fonoaudiologia da Universidade Federal de Minas Gerais - UFMG - Belo Horizonte (MG), Brasil.

Endereço para correspondência: Iara Barreto Bassi. Av. Bernardo de Vasconcelos, 2600/306, Bairro Ipiranga, Belo Horizonte - MG, CEP: 31160-440.

E-mail: iara.bassi@hotmail.com

Recebido em: 19/5/2008; Aceito em: 3/1/2009 visualização e mensuração do escape aéreo nasal, sendo também empregado como auxiliar ao diagnóstico de obstrução mecânica ou desuso nasal. Na avaliação da respiração por meio do espelho de Glatzel, observa-se a área embaçada e a simetria da quantidade de ar expelida por cada narina. Já para análise durante a fonação, o instrumento serve como auxiliar no monitoramento do escape de ar em fonemas ${ }^{(2)}$.

Embora existam exames mais confiáveis como a rinomanometria $^{(3)} \mathrm{e}$ a rinometria acústica ${ }^{(4)}$, o espelho é frequentemente empregado na avaliação fonoaudiológica, uma vez que apresenta facilidade de manuseio e baixo custo. Há, também, na literatura relatos da utilização do instrumento em avaliações otorrinolaringológicas ${ }^{(5-6)}$.

Vários estudos citam o emprego do espelho de Glatzel na avaliação da nasalidade durante a fala ${ }^{(2,7-12)}$ e da aeração nasal durante a respiração ${ }^{(6,13-15)}$. O instrumento também é utilizado na comparação do fluxo nasal do mesmo indivíduo antes e após alguma intervenção ${ }^{(14,16-17)}$. A eficácia do espelho de Glatzel só foi verificada nos aspectos referentes à fala ${ }^{(11-12)}$. Também apenas em relação à fala identificou-se na literatura parâmetros de avaliação, podendo este ser por níveis ${ }^{(2,7,9)}$ ou por pontos ${ }^{(10,11)}$.

Apesar de amplamente empregado na prática clínica, até os dias atuais não foram conduzidos estudos que verificassem a eficácia do instrumento como método diagnóstico da respiração. Assim, a mensuração do escape aéreo nasal, realizada 
na clínica fonoaudiológica pode não ser confiável e indicativa de uma obstrução nasal significativa.

Desta forma, este estudo teve como objetivo comparar a medida do escape aéreo nasal, realizada por meio espelho de Glatzel, entre crianças com e sem obstrução de vias aéreas superiores de acordo com o sexo e a faixa etária.

\section{MÉTODOS}

Foi realizado um estudo transversal, com uma amostra composta por 43 crianças, sendo $14(32,6 \%)$ do sexo feminino e $29(67,4 \%)$ do masculino, com idades variando entre quatro e 11 anos (média de 6,9 anos), dos quais $23(53,5 \%)$ apresentavam de quatro a seis anos de idade e $20(46,5 \%)$ de sete a 11 anos. Os pacientes se encontravam em atendimento no Ambulatório do Respirador Oral do Hospital das Clínicas da Universidade Federal de Minas Gerais.

As crianças foram separadas em dois grupos: participantes com obstrução nasal e controle sem obstrução. $\mathrm{O}$ grupo de crianças com obstrução nasal foi subdividido, levando-se em consideração a causa da obstrução. Assim, foram formados três grupos distintos: casos cirúrgicos $(n=7)$, casos alérgicos com manifestação de obstrução $(\mathrm{n}=11)$ e casos cirúrgicos e concomitantemente alérgicos $(\mathrm{n}=10)$.

O Grupo Controle ( $\mathrm{n}=15)$, sem obstrução, foi constituído por pacientes que eram respiradores orais cirúrgicos, não alérgicos e que foram submetidos ao procedimento com sucesso. Esses pacientes foram acompanhados clinicamente no Ambulatório do Respirador Oral por um período mínimo de três meses, sendo classificados como respiradores nasais, sem necessidade de fonoterapia para restabelecer o modo respiratório. A opção por um Grupo Controle de ex-respiradores orais decorreu do fato de se poder ter certeza de que as vias aéreas superiores encontravam-se totalmente liberadas e em uso.

Para a realização do diagnóstico os participantes foram submetidos à avaliação otorrinolaringológica, alergológica, fonoaudiológica e ortodôntica e aos exames de nasofibrolaringoscopia e teste cutâneo.

Para o grupo de crianças com obstrução, foi considerado como critério de inclusão o participante ser respirador oral obstrutivo. Para o Grupo Controle, considerou-se como critério de inclusão a presença de vias aéreas superiores totalmente liberadas e respiração exclusivamente nasal no pós cirúrgico. Estabeleceu-se como critério de exclusão para ambos os grupos, a presença de gripes, resfriados ou processos infecciosos na data da coleta.

As crianças, selecionadas aleatoriamente, foram avaliadas no Ambulatório do Respirador Oral do Hospital das Clínicas da Universidade Federal de Minas Gerais no período de março a julho de 2007. Os responsáveis pelas crianças foram informados do trabalho a ser realizado, por meio do Termo de Consentimento Livre e Esclarecido.

A medida do escape aéreo nasal foi realizada por meio do Espelho Nasal Milimetrado de Altmann (Pro-Fono®), posicionado em um ângulo de $90^{\circ}$ em relação ao filtro nasal; com a criança sentada, com as costas e os pés apoiados; respirando apenas pelas narinas e com os olhos fechados. No momento da mensuração do escape aéreo nasal, o participante foi orientado a permanecer com a boca e os olhos fechados, respirando por aproximadamente dois minutos. A medida da área embaçada no espelho foi realizada somente no segundo minuto, para que a criança se habituasse ao instrumento, descartando assim a mudança da frequiência respiratória por ansiedade.

Para marcação da área embaçada no espelho foi utilizada caneta de retroprojetor; em seguida, essa medida foi transcrita para a folha de marcação do Bloco de Referência do Espelho Nasal Milimetrado de Altmann. Cada folha do bloco de referência foi escaneada, por meio de equipamento CX5900 da marca Epson ${ }^{\circledR}$, tendo sido os dados mensurados posteriormente por meio do software AutoCAD 2002 obtendo-se a medida $\mathrm{em} \mathrm{cm}^{2}$. Os dados foram analisados por meio das medidas de tendência central e dispersão e por testes de hipóteses a um nível de significância de 5\%. Inicialmente empregou-se a análise ANOVA para se verificar a presença de diferença entre os grupos; em seguida, foi aplicado o teste de comparações múltiplas de Tukey para identificar qual ou quais grupos difeririam entre si.

Essa pesquisa foi aprovada pelo Comitê de Ética em Pesquisa da Universidade Federal de Minas Gerais sob o ${ }^{\circ}$ $105 / 07$, tendo sido considerada sem risco e com necessidade de Termo de Consentimento Livre e Esclarecido.

\section{RESULTADOS}

As medidas de tendência central e dispersão, referentes ao escape aéreo nasal por grupo, são apresentadas na Tabela 1. Na Tabela 2 encontram-se os intervalos de confiança a 95\% de acordo com o teste de comparações múltiplas de Tukey para as diferenças entre as médias das medidas do escape aéreo nasal entre grupos. Neste teste, as diferenças significantes são aquelas que não incluem o valor zero no intervalo. Assim, observa-se que apenas o intervalo de confiança para a diferença entre as médias do Grupo Controle e do Grupo Alérgico e Cirúrgico apresentou diferença estatisticamente significante.

Tabela 1. Medidas do escape aéreo nasal $\mathrm{em} \mathrm{cm}^{2}$ por grupo

\begin{tabular}{lcccccc}
\hline Grupo & $\mathrm{N}$ & Média & $\mathrm{DP}$ & Mediana & Mínimo & Máximo \\
\hline Alérgico & 11 & 4,74 & 2,49 & 4,34 & 1,83 & 9 \\
Alérgico e & 10 & 3,07 & 2,71 & 2,51 & 0,26 & 8,55 \\
cirúrgico & & & & & & \\
Cirúrgico & 7 & 5,91 & 3,13 & 5,74 & 1,92 & 12,01 \\
Controle & 15 & 6,53 & 2,13 & 6,18 & 1,68 & 10,76 \\
\hline
\end{tabular}

Análise de ANOVA: $p=0,013$

Legenda: $\mathrm{N}=$ número de sujeitos; $\mathrm{DP}=$ desvio-padrão

Tabela 2. Intervalos de confiança a $95 \%$ de acordo com o teste de comparações múltiplas de Tukey para as diferenças entre as médias das medidas do escape aéreo nasal entre grupos

\begin{tabular}{lccc}
\hline & Alérgico & $\begin{array}{c}\text { Alérgico e } \\
\text { cirúrgico }\end{array}$ & Cirúrgico \\
\hline $\begin{array}{l}\text { Alérgico e } \\
\text { cirúrgico }\end{array}$ & {$[-1,291 ; 4,643]$} & & \\
Cirúrgico & {$[-4,452 ; 2,114]$} & {$[-6,191 ; 0,501]$} & \\
Controle & {$[-4,487 ; 0,904]$} & {$[-6,240 ;-0,696]$} & {$[-3,731 ; 2,486]$} \\
\hline
\end{tabular}


Tabela 3. Medidas do escape aéreo nasal $\mathrm{em}^{\mathrm{cm}^{2}}$ por sexo

\begin{tabular}{lcccccc}
\hline Sexo & $\mathrm{N}$ & Média & DP & Mediana & Mínimo & Máximo \\
\hline Feminino & 14 & 5,55 & 2,91 & 5,83 & 0,67 & 10,76 \\
Masculino & 29 & 4,98 & 2,77 & 5,07 & 0,26 & 12,01 \\
\hline Análise de ANOVA: $\mathrm{p}=0,539$ \\
Legenda: $\mathrm{N}$ = número de sujeitos; DP = desvio-padrão
\end{tabular}

Tabela 4. Medidas do escape aéreo nasal em $\mathrm{cm}^{2}$ por faixa etária

\begin{tabular}{lcccccc}
\hline Faixa etária & $\mathrm{N}$ & Média & $\mathrm{DP}$ & Mediana & Mínimo & Máximo \\
\hline$\leq 6$ & 23 & 5,23 & 2,94 & 5,16 & 0,26 & 12,01 \\
$>6$ & 20 & 5,1 & 2,7 & 2,4 & 0,67 & 10,76 \\
\hline Análise de ANOVA: $\mathrm{p}=0,881$ & \\
Legenda: $\mathrm{N}=$ número de sujeitos; $\mathrm{DP}=$ desvio-padrão
\end{tabular}

Tabela 5. Medidas do escape aéreo nasal $\mathrm{em}^{\mathrm{cm}^{2}}$ dos grupos, por sexo e faixa etária

\begin{tabular}{|c|c|c|c|c|c|c|c|c|}
\hline Grupo & $\mathrm{N}$ & Sexo & Faixa etária & Média & DP & Mediana & Mínimo & Máximo \\
\hline \multirow[t]{4}{*}{ Alérgico } & 0 & Feminino & $\leq 6$ & NA & NA & NA & NA & NA \\
\hline & 3 & Feminino & $>6$ & 4,84 & 2,9 & 3,18 & 3,15 & 8,18 \\
\hline & 5 & Masculino & $\leq 6$ & 5,42 & 2,86 & 4,35 & 1,83 & 9 \\
\hline & 3 & Masculino & $>6$ & 3,51 & 1,64 & 3,54 & 1,85 & 5,13 \\
\hline \multirow[t]{4}{*}{ Alérgico e cirúrgico } & 1 & Feminino & $\leq 6$ & 1,28 & NA & 1,28 & 1,28 & 1,28 \\
\hline & 2 & Feminino & $>6$ & 3,9 & 4,56 & 3,9 & 0,67 & 7,12 \\
\hline & 4 & Masculino & $\leq 6$ & 3,75 & 3,47 & 3,1 & 0,26 & 8,55 \\
\hline & 3 & Masculino & $>6$ & 2,19 & 0,56 & 2,48 & 1,55 & 2,54 \\
\hline \multirow[t]{4}{*}{ Cirúrgico } & 2 & Feminino & $\leq 6$ & 4,64 & 1,43 & 4,64 & 3,63 & 5,65 \\
\hline & 0 & Feminino & $>6$ & NA & NA & NA & NA & NA \\
\hline & 3 & Masculino & $\leq 6$ & 6,59 & 5,09 & 5,85 & 1,92 & 12,01 \\
\hline & 2 & Masculino & $>6$ & 6,16 & 0,59 & 6,16 & 5,74 & 6,57 \\
\hline \multirow[t]{4}{*}{ Controle } & 3 & Feminino & $\leq 6$ & 6,4 & 0,55 & 6,18 & 5,99 & 7,03 \\
\hline & 3 & Feminino & $>6$ & 8,54 & 2,61 & 9,2 & 5,67 & 10,76 \\
\hline & 5 & Masculino & $\leq 6$ & 5,71 & 2,77 & 5,16 & 1,68 & 8,59 \\
\hline & 4 & Masculino & $>6$ & 6,15 & 0,88 & 6,16 & 5,07 & 7,23 \\
\hline
\end{tabular}

Legenda: $\mathrm{N}=$ número de sujeitos; $\mathrm{DP}$ = desvio-padrão; $\mathrm{NA}=$ não se aplica

As medidas do escape aéreo nasal dos grupos, por sexo e faixa etária, além da análise estatística podem ser observadas nas Tabelas 3 a 5 .

\section{DISCUSSÃO}

A média e mediana do Grupo Alérgico e Cirúrgico foram inferiores aos dos demais grupos, indicando valores menores de medida aérea nasal. A possível diferença indicada pelas análises descritivas foi comprovada pelos testes de hipóteses, uma vez que apenas o intervalo de confiança para a diferença entre as médias do Grupo Controle e Grupo Alérgico e Cirúrgico não contém o valor zero. Assim, apenas para estes dois grupos a diferença entre médias foi estatisticamente significante. A estimativa de tal diferença é 3,44, ou seja, o Grupo Alérgico e Cirúrgico apresenta valor de medida de escape, em média, 3,44 menor que o Grupo Controle.

Apesar de não ter sido constatada diferença entre pacientes com indicação cirúrgica para desobstrução de via aérea superior (Grupo Cirúrgico) e o Grupo Controle, na literatura pesquisada verificou-se que o aumento da permeabilidade nasal após a cirurgia de adenoidectomia foi confirmado pela rinometria acústica ${ }^{(4)}$. De acordo com estudos, a rinometria é o recurso mais apropriado para avaliar a permeabilidade nasal, uma vez que é capaz de detectar desde pequenas diferenças, como as decorrentes de mudança de postura ${ }^{(18)}$ até modificações maiores, como as consequentes ao uso de vasoconstrito- res ${ }^{(19)}$ ou de procedimentos cirúrgicos ${ }^{(20)}$.

Desta forma, o espelho de Glatzel, na amostra pesquisada, não foi capaz de indicar situações de obstrução de vias aéreas superiores. Apesar dos participantes apresentarem graus obstrutivos diversos, todos os integrantes do Grupo Cirúrgico apresentavam obstrução suficiente para uma indicação de procedimento cirúrgico.

$\mathrm{O}$ instrumento indicou diferenças apenas em casos de obstrução nasal importante, ou seja, quando duas alterações de vias aéreas superiores apresentam-se concomitantemente, embora haja relato da capacidade do espelho em demonstrar o efeito descongestionante da atividade física ${ }^{(14)}$, tendo sido este dado confirmado pela rinometria acústica ${ }^{(21)}$.

A comparação dos resultados do presente estudo com a literatura torna-se difícil, uma vez que dados específicos geralmente não são apresentados nas publicações ${ }^{(5-6,13-15,22)}$. Apesar de ser indicado o uso do espelho de Glatzel na avaliação miofuncional orofacial e cervical, em casos de respiração oral $^{(23)}$ ou de trauma de face ${ }^{(24)}$, não há padronização para aferição da permeabilidade nasal, podendo esta ser avaliada quanto à presença ou ausência de embaçamento ${ }^{(6,12)}$ quanto aos espaços marcados no próprio espelho ${ }^{(16)}$ ou por programa de computador ${ }^{(17)}$.

Observou-se a menor média da medida do escape aéreo no Grupo Alérgico e Cirúrgico feminino e menor que seis anos de idade, enquanto que a maior média foi verificada no Grupo Controle feminino e maior que seis anos de idade. 
Esse resultado corrobora com outro achado em que, na análise individual, o menor valor encontrado foi observado em um paciente pertencente ao Grupo Alérgico e Cirúrgico. Porém, não se esperava que o maior valor fosse observado em um paciente do Grupo Cirúrgico de seis anos de idade. Vale ressaltar que o Grupo Cirúrgico apresentou o maior desvio padrão, indicando ser o mais heterogêneo dentre os quatro grupos avaliados.

A análise das diferenças entre sexo e faixa etária foi realizada independentemente dos grupos, uma vez que, de acordo com o modelo de regressão linear, verificou-se que não existe interação entre as variáveis explicativas. Assim, a comparação entre os grupos independe do sexo ou da idade e, da mesma forma, a comparação entre os sexos ou entre as idades independe do grupo. Também se verificou que a comparação entre os sexos independe da idade.

Diante da análise dos resultados foi observado que a variável gênero não possui relevância. Na literatura pesquisada, estudos realizados com crianças de ambos os gêneros não fazem referência a tal variável ${ }^{(16-17)}$, nem mesmo os que empregaram rinometria acústica ou rinomanometria ${ }^{(4,20-21)}$. Observou-se, também, que não houve diferença entre faixas etárias. Os dados referentes aos grupos com obstrução concordam com uma pesquisa realizada com crianças, cujo resultado revelou prevalência de respiração oral elevada, sem diferença estatística entre os gêneros e a faixa etária ${ }^{(15)}$.

Os valores de mensuração observados no presente estudo discordam de outras pesquisas. A média verificada na literatura $^{(17)}$ para crianças de quatro a 11 anos, com rinite alérgica, antes da limpeza nasal é de $16,6 \mathrm{~cm}^{2}$, enquanto que o valor encontrado na presente pesquisa para crianças alérgicas menores de seis anos foi de $5,42 \mathrm{~cm}^{2}$ e para crianças de sete a 11 anos de $4,17 \mathrm{~cm}^{2}$. Outra divergência pode ser observada em estudo que descartou respiradores orais obstrutivos e verificou medidas pré-intervenção entre 10,7 e 14,1 cm² e pós-intervenção entre 18,4 e $26,5 \mathrm{~cm}^{2}$ para crianças de quatro anos e quatro anos e oito meses ${ }^{(16)}$. No presente estudo, a média dos valores encontrados no grupo controle para crianças até seis anos de idade foi de $5,97 \mathrm{~cm}^{2}$. Entretanto, cabe ressaltar que os três estudos empregam metodologias distintas.

A eficácia do emprego do espelho de Glatzel na avaliação da permeabilidade nasal é ainda pouco estudada, sendo raros os trabalhos que mensuram numericamente a área embaçada ${ }^{(16-17)}$. Além de escassos, como os estudos empregam metodologias diversificadas, torna-se difícil a determinação de parâmetros de mensuração que possam ser empregados na prática fonoaudiológica. Assim, seria necessário que fossem conduzidas mais pesquisas com amostras significativas para verificação do instrumento e normalização de valores da população sem alteração de via aérea superior, desenvolvendo assim uma padronização.

Para o atual estudo, a continuidade da coleta seria válida para aumentar o número da amostra estudada e, com isso, tornar os resultados mais confiáveis. Com uma amostra maior, o grupo de pacientes cirúrgicos poderia ser subdividido por graus de obstrução, eliminando um possível viés da presente pesquisa.

\section{CONCLUSÃO}

O espelho de Glatzel não demonstrou ser um instrumento confiável para a avaliação da permeabilidade nasal na amostra analisada, exceto nos casos de grande obstrução nasal, ou seja, pacientes cirúrgicos e também alérgicos. Os dados encontrados não apresentaram correlação com o sexo ou com a faixa etária.

\begin{abstract}
Purpose: To compare the measurement of nasal air escape, carried out using the Glatzel mirror, in children with and without upper respiratory tract obstruction, according to sex and age. Methods: The sample comprised 43 subjects, with mean age of 6.9 years, divided into four groups: children with surgical indication to clearing of the upper respiratory tract (surgical group), allergic children with upper respiratory tract obstruction (allergic group), allergic children with obstruction and surgical indication (surgical and allergic group), and children without obstruction (control group). Nasal air escape measurement was carried out using the Altmann's Millimetered Nasal Mirror, and data was measured in $\mathrm{cm}^{2}$ by the AutoCAD 2002 software. Data was analyzed using descriptive statistics and hypotheses testing, with a significance level of 5\%. Results: The control group presented the highest mean $\left(6.53 \mathrm{~cm}^{2}\right)$ of nasal air escape, while surgical and allergic group presented the lowest mean $\left(3.07 \mathrm{~cm}^{2}\right)$. The means of the allergic group and the surgical group were $4.74 \mathrm{~cm}^{2}$ and $5.91 \mathrm{~cm}^{2}$, respectively. Only the surgical and allergic group presented statistically significant difference compared to the control group. Conclusion: The Glatzel mirror demonstrated not to be a trustworthy instrument for the evaluation of nasal permeability in the analyzed sample, except in cases of severe nasal obstruction, that is, allergic patients with surgical indication. No correlations were found with the variables sex and age.
\end{abstract}

Keywords: Nasal obstruction; Mouth breathing; Evaluation studies; Permeability; Child

\title{
REFERÊNCIAS
}

1. Hungria H. Otorrinolaringologia. 8a ed. Rio de Janeiro: Guanabara Koogan; 2000.
2. Altmann EBC, Khoury RBF, Ramos ALNF. Avaliação fonoaudiológica. In: Altmann EBC. Fissuras labiopalatinas. 4a ed. Carapicuiba: Pró-Fono; 1997. p. 325-66. 
3. Paiva JB, Vigorito JW, Castro ABBAT. Estudo rinomanométrico da cavidade nasal em pacientes submetidos à expansão rápida da maxila. Ortodontia. 2000;33(1):36-42.

4. Nigro CEN, Goto E, Nigro JFA, M Junior JF, Mion O, Voegels RL. Avaliação da cavidade nasal e nasofaringe através da rinometria acústica antes e após adenoidectomia. Rev Bras Otorrinolaringol. 2003;69(3):333-6.

5. Silva L, Bussoloti Filho I, Paes RP, Chiattone CS, Silva AE, Brunelli LF. Linfoma de Burkitt primário de amígdalas palatinas - Relato de dois casos. Rev Bras Otorrinolaringol. 1999;65(5):450-4.

6. Simons ME, Granato L, Oliveira RCB, Alcantara MPA. Rinoscleroma: relato de caso. Rev Bras Otorrinolaringol. 2006;72(4):568-71.

7. Van Lierde KM, Van Borsel J, Moerman M, Van Cauwenberge P. Nasalance, nasality, voice, and articulation after uvulopalatopharyngoplasty. Laryngoscope. 2002;112(5):873-8.

8. Johns DF, Rohrich RJ, Awada M. Velopharyngeal incompetence: a guide for clinical evaluation. Plast Reconstr Surg. 2003;112(7):1890-7; quiz $1898,1982$.

9. Van Lierde KM, Claeys S, De Bodt M, Van Cauwenberge P. Outcome of laryngeal and velopharyngeal biofeedback treatment in children and young adults: a pilot study. J Voice. 2004;18(1):97-106.

10. Trindade IEK, Genaro KF, Yamashita RP, Miguel HC, Fukushiro AP. Proposta de classificação da função velofaríngea na avaliação perceptivo-auditiva da fala. Pró-Fono. 2005;17(2):259-62.

11. Garbino JF, Genaro KF. Concordância interjuízes na avaliação do escape nasal de ar [resumo]. Rev Soc Bras Fonoaudiol. 2006; Supl Especial.

12. Penido FA, Noronha RMS, Caetano KI, Jesus MSV, Di Ninno CQMS, Britto ATBO. Correlação entre os achados do teste de emissão de ar nasal e da nasofaringoscopia em pacientes com fissura labiopalatina operada. Rev Soc Bras Fonoaudiol. 2007;12(2):126-34.

13. Sleiman DA. Atuação fonoaudiológica nas alterações miofuncionais orais em indivíduos com maloclusão classe II. J Bras Fonoaudiol. 1999;1(1):72-8.

14. Calliari DS, Brescovici S, Kruse G. O espelho de Glatzel na avaliação da permeabilidade nasal antes e após exercício físico em indivíduos atletas [resumo]. Rev Soc Bras Fonoaudiol. 2005; Supl Especial.
15. Menezes VA, Leal RB, Pessoa RS, Pontes RMES. Prevalência e fatores associados à respiração oral em escolares participantes do projeto Santo Amaro-Recife, 2005. Rev Bras Otorrinolaringol. 2006;72(3):394-9.

16. Degan VV, Puppin-Rontani RM. Aumento da aeração nasal após remoção de hábitos de sucção e terapia miofuncional. Rev CEFAC. 2007;9(1):55-60.

17. Melo FMG, Cunha DA, Silva HJ. Avaliação da aeração nasal pré e pós a realização de manobras e massagens de limpeza nasal. Rev CEFAC. 2007;9(3):375-82

18. Roithmann R, Demeneghi P, Faggiano R, Cury A. Efeitos da alteração de postura sobre a permeabilidade nasal. Rev Bras Otorrinolaringol. 2005;71(4):478-84.

19. Zancanella E, Lima WTA. Uso da rinometria acústica como método diagnóstico. Rev Bras Otorrinolaringol. 2004;70(4):500-3.

20. Santos RP, Habermann W, Hofmann T, Stammberger H. Avaliação do volume da cavidade nasal por rinometria acústica, pré e pós-cirurgia endoscópica funcional dos seios paranasais. Rev Bras Otorrinolaringol. 2006;72(4):549-53

21. Fonseca MT, Machado JAP, Pereira SA, Pinto KM, Voegels RL. Efeito do exercício físico sobre o volume nasal. Rev Bras Otorrinolaringol. 2006;72(2):256-60

22. Trindade IEK, Gomes AOC, Sampaio-Teixeira ACM, Trindade SHK. Volumes nasais de adultos aferidos por rinometria acústica. Rev Bras Otorrinolaringol. 2007;73(1):32-9.

23. Marquesan IQ. Protocolo de avaliação miofuncional orofacial. In: Krakauer LH, Di Francesco RC, Marchesan IQ, organizadores. Conhecimentos essenciais para entender bem a respiração oral: abordagem interdisciplinar. São José dos Campos: Pulso Editorial; c2003. p.55-79.

24. Bianchini EMG. Traumas de face: alteração fonoaudiológica, caracterização, proposta terapêutica e resultado. In: Comitê de Motricidade Orofacial da Sociedade Brasileira de Fonoaudiologia. Motricidade orofacial: como atuam os especialistas. São José dos Campos: Pulso Editorial; 2004. p.107-14. 